\title{
Chronic periaortitis and antiphospholipid syndrome: is there a link?
}

\author{
Carneiro $L^{1}$, Ferreira $A^{1}$, Rios $E^{2,3,4}$ \\ ${ }^{1}$ Medicine Department, Hospital Pedro Hispano, Matosinhos, Portugal \\ 2 Pathology Department, Centro Hospitalar de São João, Porto, Portugal \\ ${ }^{3}$ Pathology and Oncology Department, Medical Faculty of the University of Porto, Porto, Portugal \\ ${ }^{4}$ Institute of Molecular Pathology and Immunology of the University of Porto (IPATIMUP), Porto, Portugal
}

\begin{abstract}
Chronic periaortitis (CP) is a rare fibro-inflammatory disease characterized by periaortic fibrosis and/or aortic aneurysms formation, mostly localized in retroperitoneum and occasionally in the mediastinum. Recent studies have shown its common association with autoimmune diseases, therefore autoimmunity has been proposed as a contributing factor.

Herein, we describe the second case in the literature of CP associated with antiphospholipid syndrome. A 64-year-old man with history of open surgery for inflammatory thoracic aortic aneurysm and recurrent deep vein thrombosis was referred for abdominal pain and weight loss in the last 6 months. Further investigation revealed elevated acute-phase reactant levels, positive antiphospholipid autoantibodies, radiological and histological evidence of periaortic fibrosis and inflammation causing abdominal aortic aneurysm and ureteral obstruction. Diagnosis of CP and antiphospholipid syndrome were made and steroid therapy was implemented with clinical and radiological improvement. The present report further supports the potentially immune-mediated origin of $\mathrm{CP}$, highlighting its possible linkage with antiphospholipid syndrome.
\end{abstract}

Keywords: Periaortitis, Chronic; Antibodies, Antiphospholipid; Aneurysm.

Palabras clave: Periaortitis, Crónica; Anticuerpos, antifosfolípido; Aneurisma.

\section{Introduction}

Chronic periaortitis (CP) is a rare idiopathic disease, characterized by a thickening of the aortic wall which may vary from an inflammatory infiltrate in the acute phase until a fibrous scar in more advanced stages and usually manifests by symptoms resulting from compression of surrounding structures, especially, urinary tract and vascular structures ${ }^{[1-3]}$.CP encompasses three main entities namely: idiopathic retroperitoneal fibrosis (IRF); inflammatory aortic aneurysms (IAAs) and perianeurysmal retroperitoneal fibrosis (PRF). In IAAs and PRF the fibroinflammatory tissue develops around a dilated aorta, and consequently are referred to as aneurysmal forms of CP. IRF corresponds to the non-aneurysmal form ${ }^{[1-4]}$.

Current theories suggest two potential pathogenetic mechanisms of CP. Initially considered as a localized inflammatory response to advanced aortic atherosclerosis, recent studies have demonstrated that $\mathrm{CP}$ behaves more like an auto-immune disease, leading to the assumption that an underlying autoimmune mechanism might explain the pathogenesis of CP. This concept is supported by its frequent association with autoimmune diseases including ankylosing spondylitis, rheumatoid arthritis (RA) and systemic lupus erythematosus (SLE). Nevertheless, to our knowledge, there is only one previously reported case of concurrent $\mathrm{CP}$ and antiphospholipid syndrome (APS) ${ }^{[5]}$.

This review will outline the relevant aspects of clinical presentation and diagnosis, therapy and prognosis of CP and will particularly discuss the different proposed immunological mechanisms, suggested as responsible for this association.

\section{Clinical case}

A 64-year-old man was referred to internal medicine consultation with a 6-month history of anorexia, weight loss (22\% of body weight) and progressive pain in the abdomen radiating to the lower back. He had a past strong medical history of cardiovascular disease with systemic hypertension, coronary disease and open surgical repair of a ruptured ascending thoracic aortic aneurysm 4 years before. Relevant medical background also included two episodes of deep venous thrombosis (DVT) in the last year. On physical examination there was an abdominal pulsatile mass. Initial laboratory findings showed microcytic anemia (8.9 g/dL; N 12-13 $\mathrm{g} / \mathrm{dL})$, elevated erythrocyte sedimentation rate $(133 \mathrm{~mm} 1 \mathrm{st}$ hour; $\mathrm{N}<20 \mathrm{~mm} 1 \mathrm{st}$ hour) and C-reactive protein (13 mg/dL; $\mathrm{N}<5 \mathrm{mg} / \mathrm{L})$, and renal dysfunction (creatinine $2.8 \mathrm{mg} / \mathrm{dL} ; \mathrm{N}<1 \mathrm{mg} / \mathrm{dL}$ ) without proteinuria or red blood cells casts. The serum proteinogram showed polyclonal hypergammaglobulinemia with raised level of lgG (1945 mg/dl) but normal serum IgG4 concentrations. Serological testing for EBV, CMV, HSV, parvovirus B19, HIV, hepatitis B and C, syphilis and tuberculosis skin testing were negative. Immunological studies revealed persistent high levels of IgM anticardiolipin (aCL) antibody (159.5 MPL, positive >11) and IgM $\beta 2$-glycoprotein (B2G) antibody (168 U/mL, positive $>11)$, with negative lupus anticoagulant, measured on two occasions 4 months apart. Workup for other autoimmune disorders was unremarkable, including negative anti-neutrophil cytoplasmic antibodies and antinuclear antibody panel. A thoracoabdominal computed tomography (CT) scan was made and showed an aneurysm of the infrarenal abdominal aorta with $4.1 \mathrm{~cm}$ in diameter and bilateral hydronephrosis (figure 1 - C). In addition, there was a 'sleeve' of abnormal material of soft tissue density surrounding the right brachiocephalic trunk, aortic arch, descending aorta, abdominal aorta and common iliac arteries (Figure 1 - A and 1-B). Positron emission tomography (PET) re- 
vealed increased pathologic activity at the level of ascending aorta (Figure 1 - D). Endoscopic study of gastrointestinal tract showed multiple angiectasis in the colon with signs of recent bleeding that were treated with argon plasma coagulation. To confirm the diagnosis of CP but also to exclude other diseases such as neoplasia and infections, a biopsy of retroperitoneal tissue was done. Histopathology showed a dense lymphoplasmacytic infiltrate and there were no signs of malignancy. Histopathological review of the surgical specimen obtained from previous surgery on the thoracic aorta (Figure 2-A) revealed a markedly thickened adventitia due to severe fibrous proliferation with lymphocytes (occasionally lymphoid follicles), plasma cells and focal microcalcifications (Figure 2-B and 2-C). Atherosclerotic intimal change was also observed. The immunohistochemical study detected only a few lgG4-positive plasma cells in the adventitia (Figure 2-D).

A double $\mathrm{J}$ catheter insertion was performed for bilateral hydronephrosis and prednisolone at an initial dose of $1 \mathrm{mg} / \mathrm{kg}$ per day (80 $\mathrm{mg} /$ day) was started, with marked clinical improvement and rapid normalization of systemic markers of inflammation. Based on the clinical presentation of DVT and elevated titers of aCL and B2G antibodies the diagnosis of APS was established. Given the presence of moderate anemia and high risk of bleeding it was decided not to initiate anticoagulation and maintain only a low-dose of aspirin. At present time the patient completed 6 months of treatment and is now on a maintenance dose of $10 \mathrm{mg} /$ day of prednisolone with resolution of clinical symptoms and inflammatory markers. The 6-month-follow up thoracoabdominal CT scan showed a slight reduction of fibrotic periaortic sleeve and persistent dilatation of the urinary system. Regular aneurysm surveillance revealed dimensional stability.

\section{Discussion}

Recent studies have shown that CP may also involve other vascular segments, such as the coronary, renal and mesenteric arteries ${ }^{[6-10]}$ and represent, at least in some patients, a systemic vascular process, rather than a localized inflammatory reaction ${ }^{[6]}$. The presentation of aortic involvement may range from inflammatory aneurism with or without perianeurysmal fibrosis to isolated periaortic (retroperitoneal and/or mediastinal) fibrosis with compression of the aorta ${ }^{[6-9]}$.

This patient had an aneurysmal form of CP (PRF) with extensive thoracic and abdominal periaortic involvement, which is extremely uncommon. As a consequence, the inflammatory aneurysms associated with perianeurysmal retroperitoneal fibrosis had already leaded to bilateral hydronephrosis and rupture of ascending thoracic aneurysm 4 years before.

The pathogenesis of CP is still unclear. Initially, the leading theory was that CP was a consequence of a local autoimmune reaction against atherosclerotic plaque antigens ${ }^{[1-3}$, ${ }^{6,10]}$. However, the absence of atherosclerosis in some patients, and the presence of constitutional symptoms, good

Figure 1. Thoracoabdominal CT scan contrast - enhanced CT scan (axial views) showing non-smooth thickening of soft tissue around aortic arch (A, arrow) and abdominal aorta (B, arrow). The abdominal aorta shows aneurysmal dilatation (C, arrow). Positron emission tomography showing increase ${ }^{18}$ Fluorodeoxyglucose uptake in the ascending aorta ( $\mathbf{D}$, arrow).
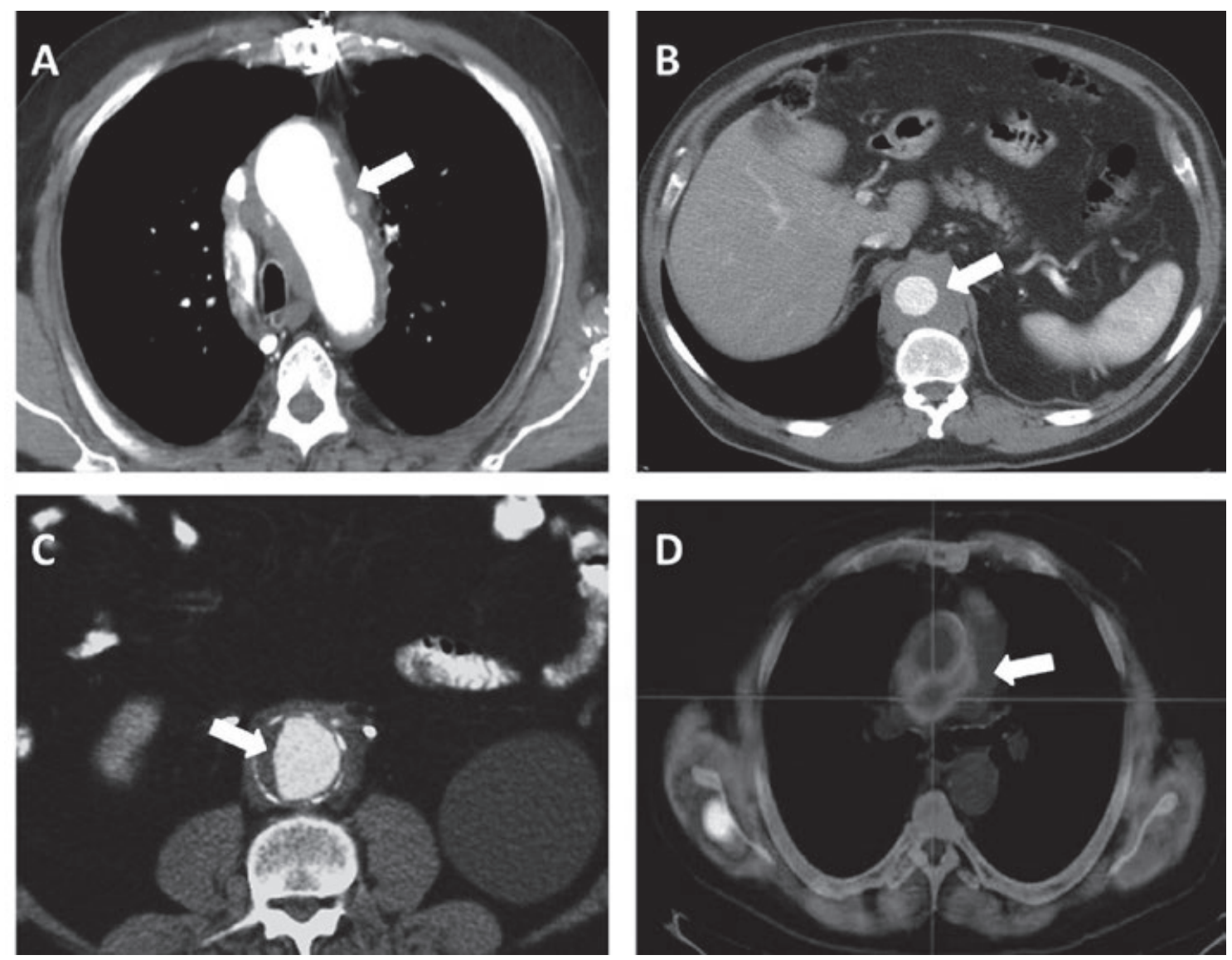
Figure 2. Anatomopathological findings of inflammatory thoracic aortic aneurysm: (A) grossly there are partial significant thickening $(1.7 \mathrm{~cm})$ of the adventitia (arrow) contrasting with the non-thickened wall (asterisk). These features are illustrated on histology (H\&E, x0.3) (B) that shows a fibrous thickening adventitia (arrow) contrasting with the non-thickened wall (asterisk). Lymphoplasmacytic infiltration and sclerosis are shown in the adventitia at higher magnification (H\&E, X40) (C). Only a few lgG4-positive plasma cells are detected in the adventitia (IgG4 stain, X400) (D).

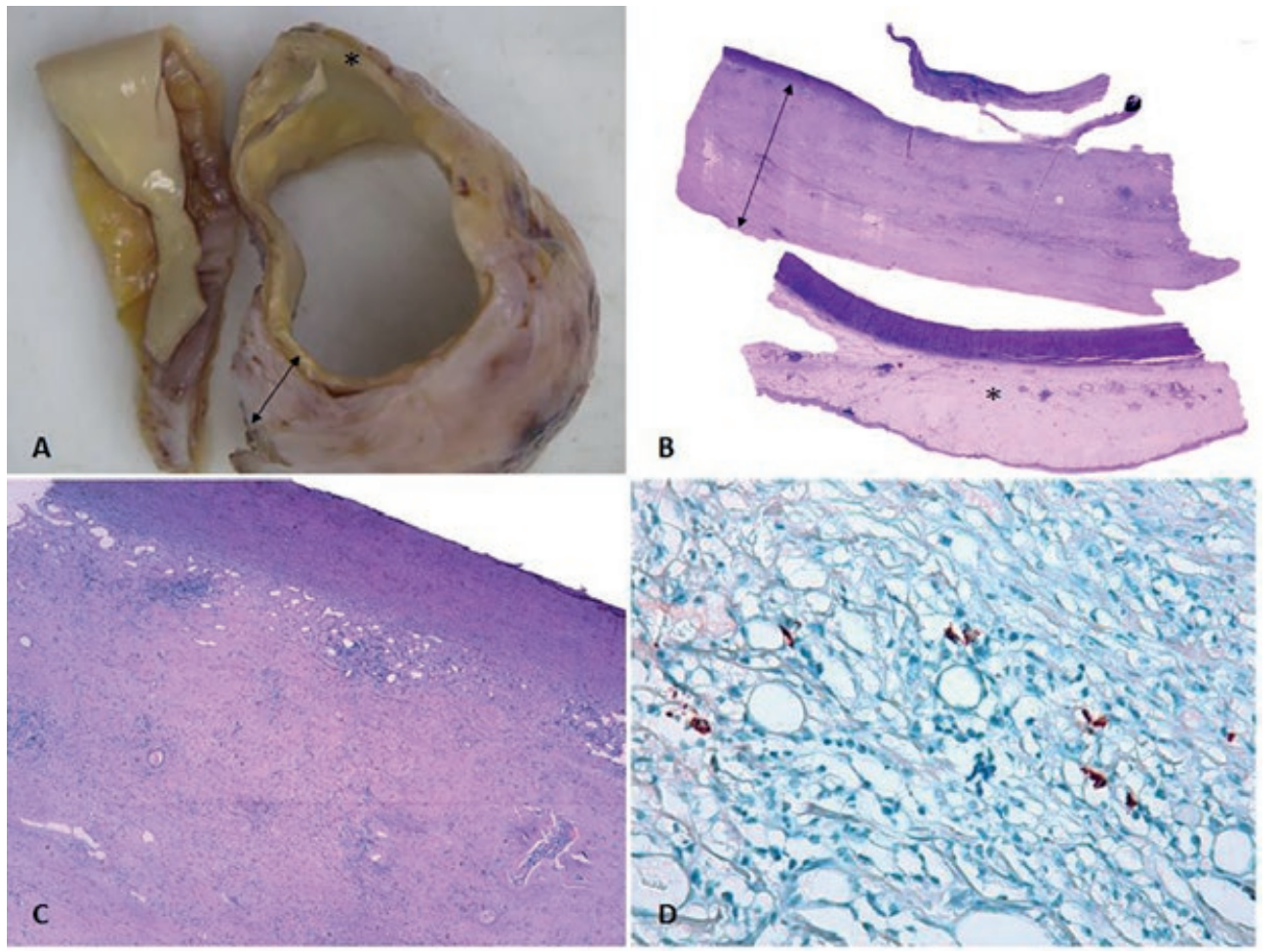

response to immunosuppression, coexistence with other autoimmune conditions including SLE and granulomatosis with polyangiitis, suggested a systemic immune-mediated process. Additionally, histopathological findings of putative vasculitis (with fibrinoid necrosis) involving the aortic vasa vasorum have been reported[6], suggesting that $\mathrm{CP}$ could be a primary aortitis. The hypothesis of an immunological basis is further supported by the higher prevalence of HLADRB1*03 allele and CC chemokine receptor 5 (CCR5) gene D32 polymorphism in patients with $\mathrm{CP}$, conferring susceptibility to the development of an exuberant cellular and humoral immune response to antigenic stimuli and subsequent progression to fibrosisi ${ }^{[11,12]}$. Taken together, these findings support the concept of immune-mediated disorder.

Although the strong cardiovascular background of our patient supported the initial hypothesis that CP is a localized inflammatory response to aortic atherosclerosis, such association may simply occur because atherosclerosis commonly affecting middle-aged to elderly persons. The presence of an underlying autoimmune disease, such as APS, further supports the hypothesis that $\mathrm{CP}$, which is considered to be idiopathic in most cases, is related to an autoimmune systemic disease. Our case seems to demonstrate that autoimmunity plays an active role in the pathogenesis of these two apparently different clinical entities, highlighting a possible linkage between them.

Additionally, the presence of constitutional symptoms along with elevated systemic inflammatory markers and in- creased uptake in the ascending thoracic aorta at $18 \mathrm{~F}$-fluorodeoxyglucose positron emission tomography (FDG-PET) scan further supports the concept that $\mathrm{CP}$ is rather a systemic process.

Finally, several recent reports have shown that CP may also display the same clinicopathological features of IgG4-related systemic diseases (IgG4-RSD), characterized by diffuse organ infiltration by lgG4-expressing plasma cells and increased serum levels of $\lg G 4^{[13,14]}$. Since our patient had only a few IgG4-expressing plasma cells on histology and normal serum IgG4 concentrations, diagnosis of an lgG4- related periaortitis was excluded.

Disease symptoms are vague and nonspecific and can make diagnosis difficult. The early phase of the disease is of variable duration, being lumbar and abdominal pain the most common symptoms. They may be associated with weight loss, anorexia, nausea, vomiting and malaise. Renal injury is often the result of obstructive uropathy caused by ureteral encasement, but vasculitic involvement may also be present in some cases ${ }^{[15]}$. There is rarely extent of fibrosis to the duodenum, bile ducts, pancreas, colon, epidural space, with very different clinical presentations.

The positivity of anti-neutrophil cytoplasmic antibodies, rheumatoid factor and antibodies against smooth muscle cells and thyroglobulin, detected in some patients, may indicate an associated auto-immune disorder ${ }^{[1,2,5,6]}$.

Definitive diagnostic tests include contrast-enhanced CT scan and magnetic resonance imaging (MRI). Lately the 
FDG-PET scan has been used for assessing the metabolic activity of the periaortic inflammatory tissue.

Although of an overall good prognosis, CP may follow a chronic persistent or relapsing course in 10 to $50 \%$ of patients and may require long-term immunosuppressant therapy. Steroids are considered the first-choice option in medical treatment reflecting the autoimmune mechanisms in disease pathogenesis. Other immunosuppressive drugs, such as azathioprine, cyclosporine, mycophenolate mofetil and methotrexate have been used as steroid-sparing agent or in those patients not responding to steroids alone. Treatment decisions related to the degree of dose reduction must be individualized and based on clinical and radiological response. Other drugs with different mechanism of action, such as tamoxifen, have recently been used as adjuvant in steroids treatment with apparent success ${ }^{[1-3,15]}$. In the early stages of disease, when the inflammatory component is more important than the fibrotic component, placement of ureteral stent or nephrostomy followed by medical treatment is recommended, without recourse to surgery ${ }^{[2,15]}$. In the aneurysmal forms of $\mathrm{CP}$, as in our case, the aim of aneurysm treatment is to prevent rupture. Therefore, surgical treatment appears prudent once the diameter of the aneurysm exceeds $5.5 \mathrm{~cm}$. In some cases, the inflammatory process may persist or even progress despite surgery ${ }^{[2]}$.Diagnosis of persistent CP following surgical aneurysm exclusion was also taken in our case, as PET scan showed persistence of metabolic activity in ascending aorta. Due to chronic and relapsing nature, long-term follow-up is recommended in all patients.

Herein we illustrate the importance of the awareness and early recognition of $\mathrm{CP}$ in order to prevent the severe secondary complications of renal failure (due to ureteral obstruction) and the potentially fatal consequence of aortic rupture. We also report, as far as we know, the second case of CP associated with an antiphospholipid syndrome, which lead us to the conclusion that autoimmunity has an active role in the CP pathogenesis. Further studies are needed to understand the relationship between these entities.

\section{References}

1. Chang-Hee S. Chronic Periaortitis as a Systemic Autoimmune Disease, Autoimmune Disorders - Pathogenetic Aspects, Prof. Mavragani CP (Ed.), InTech, 2011:303-320.

2. Vaglio A, Greco P, Corradi D, Palmisano A, Martorana D, Ronda N, Buzio C. Autoimmune aspects of chronic periaortitis. Autoimmun Rev 2006; 5:458-464.

3. Vaglio A, Buzio C. Chronic periaortitis: a spectrum of diseases. Curr Opin Rheumatol 2005; $17: 34-40$

4. Vaglio A, Salvarani C, Buzio C. Retroperitoneal fibrosis. Lancet 2006; 367:241-251.

5. Kim HA, Won JH, Suh $\mathrm{CH}$. Chronic periaortitis with antiphospholipid syndrome. Int J Rheum Dis.2010; 13:91-3

6. Vaglio A, Pipitone N, Salvarani C. Chronic periaortitis: a large-vessel vasculitis? Curr Opin Rheumatol. 2011; 23:1-6.

7. Crawford JL, Stowe CL, Safi HJ, Hallman CH, Crawford ES. Inflammatory aneurysms of the aorta. J Vasc Surg 1985; 2:113-124.

8. Okita Y, Takamoto S, Ando M, Kawashima Y, Matsuo H. A case report of inflammatory aneurysm of the thoracic aorta. J Vasc Surg 1995; 21:999-1001.

9. Roth M, Lemke P, Bohle RM, Klovekorn WP, Bauer EP. Inflammatory aneurysm of the ascending thoracic aorta. J Thorac Cardiovasc Surg 2002; 123:822-824.

10. Palmisano A, Vaglio A. Chronic periaortitis: a fibroinflammatory disorder. Best Pract Res Clin Rheumatol 2009; 23:339-353.

11. Martorana D, Vaglio A, Greco P, Zanetti A, Moroni G, et al. Chronic periaortitis and HLADRB103: another clue to an autoimmune origin. Arthritis Rheum. 2006; 55:126130

12. Boiardi L, Vaglio A, Nicoli D et al. CC chemokine receptor 5 polymorphism in chronic periaortitis. Rheumatology (Oxford). 2011; 50:1025-1032.

13. Kasashima S, Zen Y. IgG4-related Inflammatory Abdominal Aortic Aneurysm, Spectrum of IgG4-related Chronic Periaortitis. Ann Vasc Dis. 2010; 3:182-189.

14. Ishizaka N, Sakamoto A, Imai Y, Terasaki F, Nagai R. Multifocal fibrosclerosis and IgG4-related disease involving the cardiovascular system. J Cardiol. 2012; 59:132-138.

15. Urban ML, Palmisano A, Nicastro M, Corradi D, Buzio C, Vaglio A. Idiopathic and secondary forms of retroperitoneal fibrosis: A diagnostic approach. Rev Med Interne. 2015; 36:15-21 